\title{
Informalidad, productividad y flexibilidad laboral
}

\author{
Nikita Céspedes Reynaga ${ }^{1}$
}

\begin{abstract}
This document studies the relationship of labor informality with productivity and labor flexibility. It is shown that both labor flexibility and productivity are negatively related to labor informality, with which we may expect less informality when productivity and labor flexibility increase. The relationship between informality and labor flexibility is non-linear, this means that the effect of labor flexibility policies is greater in economies with high labor rigidity. Furthermore, policies that enhance productivity growth may be more effective in its ability to reduce informal employment compared to labor and wage flexibility policies.
\end{abstract}

Keywords: Informality, productivity, labor flexibility, wage rigidity.

\section{RESUMEN}

En este documento se estudia la relación de la informalidad laboral con la productividad y la flexibilidad laboral utilizando datos por países. Se muestra que la flexibilidad laboral y la productividad están negativamente relacionadas con la informalidad laboral, con lo cual se sugiere que cuando la flexibilidad laboral y la productividad se incrementan se esperaría una menor informalidad laboral. La relación entre informalidad y flexibilidad laboral es no lineal, con lo cual se podría reducir la informalidad mediante una mayor flexibilización laboral, siendo este efecto mayor en las economías con alta rigidez laboral. Se encuentra, además, que la productividad es más efectiva en su capacidad de reducción de la informalidad laboral en comparación a la flexibilidad laboral.

Palabras clave: Informalidad, productividad, flexibilidad laboral, rigidez salarial.

Received: 15 de abril del 2020

Accepted: 15 de mayo de 2020

\section{Introducción}

La importancia del sector informal es indudablemente una de sus características estructurales más destacables de las economías en desarrollo. La poca reducción de la informalidad en décadas recientes en las cuales muchas de las económicas en desarrollo han experimentado un destacable crecimiento económico es también otra característica desde la perspectiva cíclica que ha caracterizado a la dinámica de la informalidad. Los factores que han sido relevantes en esta reducción es tema de un amplio debate en la literatura de la economía sobre informalidad y en el que el crecimiento económico toma un rol preponderante al igual que diversos aspectos de la regulación laboral.

Otro factor clave en la dinámica de la informalidad son las características de flexibilidad laboral y salarial del mercado laboral. Este estudio se dedica a mostrar la relevancia de la flexibilidad laboral y de la productividad como elementos que explican la dinámica de la informalidad laboral. Se argumenta que estas dos variables se complementan entre ellas, y en este estudio se evalúa si las económicas con alto crecimiento de la productividad y con bajos índices de rigidez laboral han sido más eficientes en afectar a la informalidad en las últimas décadas.

En este estudio utilizamos un indicador de informalidad utilizado por Loayza y Rigolini (2006) en un estudio comparativo a nivel internacional. No obstante, se debe recalcar que la discusión sobre la definición operativa de informalidad ha dado lugar a recomendaciones de la Organización Internacional del Trabajo (OIT) que ha propiciado la construcción de series más ricas en términos de la identificación del sector informal, sin embargo, estas series aún no tienen la cobertura internacional para ser utilizado estudios comparativos. En este contexto, brevemente mencionamos que el sector informal se define como el conjunto de empresas, trabajadores y actividades que operan fuera del marco legal de la actividad económica (De Soto, 1989).

Desde una perspectiva general, la economía informal o subterránea puede definirse como aquellas actividades y agentes (empresas y trabajadores) que operan fuera de los marcos legales y no son registrados en las cuentas nacionales (Schneider, 1994, 2005). Sin embargo, dado que la informalidad es un fenómeno de naturaleza multidimensional, en la práctica existe pluralidad de definiciones operativas (OIT y WTO, 2009). En particular, la informalidad laboral puede definirse de forma genérica como la condición laboral que está fuera del sistema tributario, seguridad social y otras regulaciones (OIT, 2003; OECD, 2004).

La informalidad laboral se ha adecuado a estas definiciones generales, y similar al caso anterior, existe una diversidad de definiciones operativas. Así, por ejemplo, en Loayza y Rigolini (2006) se considera al autoempleo como un proxy

${ }^{1}$ Investigador docente de la Escuela Profesional de Economía de la Facultad de Ciencias Contables, Económicas y Financieras de la Universidad de San Martín de Porres. E-mail:ncespedesr@usmp.pe

How to cite: 1 INVESTIGADOR: Céspedes, N. (2020). Informalidad, productividad y flexibilidad laboral. Revista de Análisis Económico y Financiero, Vol.3,N.1, 1-6. DOI: 10.15446/ing.investig.xxxx 
de informalidad para estudiar la influencia de la actividad económica sobre esta.*

La relación de la informalidad con la flexibilidad laboral ha sido poco estudiada formalmente, aunque existe una amplia literatura que documenta la relación de la informalidad con los costos relativos asociados a la formalidad de los negocios. Así, por ejemplo, Loayza y Rigolini (2006) encuentran que la actividad económica tiene una significativa relación con la informalidad laboral (Figura 1), además, se reporta que la informalidad es mayor en aquellos paises que reportan poca flexibilidad para los negocios, cuando la estructura legal no es favorable y cuando los recursos del Estado son escasos.

\section{FIGURA 1}

Este documento complementa el estudio de Loayza y Rigolini (2006) al incorporar de manera conjunta indicadores de flexibilidad laboral y de productividad como variables que afectan a la informalidad. Se considera dos indicadores de flexibilidad laboral como la facilidad para contratar y despedir trabajadores y la flexibilidad salarial, ambos estimados por World Economic Forum (WEF). Estos datos se encuentran disponibles para diversas economías del mundo desde el año 2007 con frecuencia anual. Notar que la incorporación de la productividad y de la flexibilidad laboral de manera conjunta puede dar elementos de discusión sobre la importancia relativa de cada uno de estos indicadores como determinantes de la informalidad.

Este último aspecto parece haber sido poco estudiado en la literatura, y por lo tanto este estudio explora la posibilidad de que los dos tipos de determinantes de la informalidad mencionados se puedan reforzar y/o complementar en su capacidad de reducción de la informalidad. En la Figura 2 muestra que en los datos se reporta una alta correlación entre la productividad y la informalidad laboral a nivel mundial.

\section{FIGURA 2}

Se sugiere, además, que la relación entre informalidad y flexibilidad laboral podría ser no lineal, esto bajo la premisa que en economías con baja rigidez laboral, cambios en este pueden no tener efectos sobre la informalidad laboral, mientras que cuando la rigidez es alta esta esta podría ser más restrictiva y tener efectos mayores sobre la informalidad. Una primera inspección de los datos sugiere que la informalidad y rigidez laboral se encuentran negativamente relacionados cuando se permite esta no linealidad, es decir cuando se utiliza a la interacción del indicador de flexibilidad laboral y el PBI per cápita como variable que se correlaciona con la informalidad.

Se muestra, además, que la informalidad y la flexibilidad laboral estarian relacionadas, con lo cual aquellos paises con alta rigidez laboral coinciden en tener altos niveles de informalidad. Resultados simulares se reportan cuando se incorpora a la interacción de la productividad con los indicadores de flexibilidad laboral.

El documento utiliza un enfoque básicamente empírico similar al utilizado por Loayza y Rigolini (2006). Se considera una forma reducida que expresa a la informalidad laboral en términos de la productividad, de la actividad económica y de la productividad, conjúntame con otros elementos de control de la heterogeneidad entre países. Utilizando datos para una muestra de países se reporta que la productividad y la flexibilidad laboral tienen efectos sobre la informalidad laboral. Estos efectos, además, son no lineales en el sentido de que la flexibilidad laboral tiene efectos mayores sobre la informalidad cuando los niveles de flexibilidad son bajos (o complementariamente cuando los niveles de rigidez laboral son altos) (ver Figuras 3 y 4).

\section{FIGURA 3}

\section{FIGURA 4}

El resto del documento se organiza de la siguiente manera. La sección dos muestra el modelo empírico utilizado, la sección tres describe los datos utilizados y las fuentes de información de donde estos provienen. La sección cuatro muestra los resultados y la discusión relevante, la sección cinco resume los principales aspectos del estudio.

\section{Breve revisión de la literatura}

La literatura que relaciona a la regulación laboral con la informalidad provee de resultados mixtos y algunas veces opuestos. Se puede identificar dos vertientes de esta literatura. Por un lado, se tiene a los estudios que sugieren que la regulación laboral tiene efectos negativos en el empleo y favorece a la informalidad. Esta rama de la literatura se fundamenta básicamente en las teorías de mercado o teoría neoclásica, para quienes la regulación constituye fricciones de mercado que generan externalidades negativas en la creación de empleos formales. Evidencia de esta literatura es consistente con la hipótesis de que la excesiva legislación laboral puede ser responsable de mayor informalidad, y es destacado por el WDR (1990).

Asimismo, Perry y otros (2007) plantean que la excesiva legislación laboral pude reducir los empleos en el sector formal. Feldmann (2009) enfática que la regulación laboral demasiado estricta puede incrementar el desempleo y por los tanto la informalidad. Literatura por el lado de la demanda laboral como en Ameida and Carneiro (2009) encuentra que la estricta regulación laboral limita el tamaño de las firmas (formales) y conduce a mayor desempleo (e informalidad).

Asimismo, existe una amplia literatura sobre los efectos de la regulación sobre la informalidad utilizando datos a nivel de firmas que buscan explicar las regularidades agregadas. Considerando que la regulación puede entenderse como fricciones del mercado laboral que generan rigideces en la contratación, despido y fijación de salarios, se menciona brevemente algunos documentos relevantes de esta literatura. Además, si bien esta literatura es a nivel de empresas y el enfoque de este estudio es a nivel agregado, los documentos revisados pueden ser útiles para mostrar el mecanismo mediante al cual la regulación afecta a la informalidad.

*Otros estudios comparativos a nivel internacional consideran a la proporción de personas que tienen acceso a un sistema de pensiones como un proxy de empleo formal. 
Algunos de estos estudios recientes centrados en el impacto de la regulación de impuestos y costos de entrada en la informalidad y los resultados agregados incluyen a Rocha Ulyssea y Rachter (2018), Monteiro y Assunao (2012), Fajnzylber, Maloney y Montes-Rojas (2011), Almeida y Carneiro (2012) y De Andrade, Bruhn y McKenzie (2014).

La otra vertiente de la literatura sugiere que la legislación laboral argumenta que esta puede tener efectos favorables especialmente en los trabajadores, si bien es aceptado que la legislación (especialmente cuando esta es muy limitante) puede tener efectos negativos, es posible que los beneficios de esta legislación puedan ser mayores a los costos.

En términos de los beneficios por ejemplo se argumenta que la regulación puede tener efectos positivos en la productividad al fomentar la capacitación y la innovación (por ejemplo en Deakin y Sarkar, 2008). En términos generales, se argumenta que estos efectos pueden variar según la industria, la región y el país, y que la implementación de la ley necesariamente se verá afectada por las normas y prácticas sociales de cada caso (ILO, 2013).

Si bien la literatura es diversa en ambas direcciones, es razonable argumentar que la legislación laboral podría ser menos limitante para los empleos formales cuando esta no es tan estricta. De hecho, gran parte de la literatura que reporta efectos negativos de la regulación sobre la informalidad considera los casos de regulación estricta o de alta rigidez laboral como representativos de sus estudios.

Un aspecto que esta evidencia permite plantear, a modo de motivación, es la probable existencia de un umbral mínimo de la legislación laboral a partir del cual esta se hace costosa y fomenta una mayor informalidad laboral. Si este umbral existe ambas vertientes de la aliteratura podrían ser consistentes, esta última rama de literatura aún no ha sido tomada en consideración y se sugiere una evaluación formal.

\section{El modelo}

Se utiliza un modelo que relaciona a la informalidad laboral con la rigidez en el mercado de trabajo. Se sugiere que esta relación es no lineal y se expresa mediante la siguiente ecuación:

$$
\text { Informalidad }_{i t}=\alpha+\beta \text { Flex }_{i t}+\theta \text { Flex }_{i} t \times P B I_{i t-1}+\delta X_{i t}+v_{i t}
$$

En esta ecuación la informalidad laboral en el país i $y$ en el periodo $\mathrm{t}$ de denota por Informalidad It, la flexibilidad laboral se representa por Flexit. Se controla por la presencia de heterogeneidad en los diversos países mediante la introducción de la variable $X_{i t}$. $v_{i t}$ denota a la heterogeneidad no observable que se suponde bien comportada desde la perspectiva estadística.

En este documento se argumenta que la relación entre la flexibilidad laboral y la informalidad es no lineal. Bajo esta premisa, una mayor flexibilidad laboral podría tener efectos mínimos en la informalidad cuando la flexibilidad laboral es alta, mientras que para niveles muy bajos de flexibilidad, que podrían hacen prohibitiva la contratación y el despido de trabajadores, la informalidad podría ser la única posibilidad accesible. Bajo la premisa anterior se introduce un término cuadrático que sugiere que la flexibilidad laboral puede afectar a la informalidad y que este efecto depende del nivel y/o magnitud de la rigidez laboral.

Una segunda fuente de no linealidad se relaciona con el argumento de que la relación entre la informalidad y la flexibilidad laboral podría depender del nivel de desarrollo de los países. Estudios diversos son útiles para sustentar la no linealidad sugerida al reportar que la informalidad es mayor en aquellas economías con menores niveles de PBI per cápita lo cual justifica la inclusión del PBI per cápita (rezagado) entre este conjunto de variables de control.

En Loayza (2006), por ejemplo, se estudia la relación de la informalidad laboral con el PBI per cápita tanto en su tendencia de largo plazo como a nivel del ciclo económico. Si bien la causalidad sugerida va desde el crecimiento económico hacia la reducción de la informalidad, existe una amplia literatura que sugiere que la relación debería ir en sentido contrario, es decir la informalidad sería un limitante del crecimiento económico, especialmente del crecimiento económico de largo plazo mediante sus efectos en la productividad de la economía . Con las consideraciones anteriores se incluye un término de interacción entre la rigidez laboral y el PBI per cápita como una variable explicativa de la informalidad.

\section{Los datos}

Los datos provienen de distintas fuentes las que son adecuadamente consolidadas con fines de comparación entre países. La primera variable en consideración es la tasa de autoempleo como indicador de informalidad laboral, este indicador corresponde a la proporción de personas autoempleadas respecto a la población ocupada. La tasa de autoempleo es un indicador que está altamente correlacionada con la tasa de informalidad, y como sugiere Loayza y Rigolini (2006) esta correlación es mayor en muchas economías en desarrollo. La tasa de autoempleo se obtiene de la Organización Internacional del Trabajo, estos datos son anuales y abarcan a 89 países hasta el 2016. En estos datos la tasa promedio de autoempleo es 27,9 por ciento (Ver Tabla 1).

\section{TABLA 1}

Se utiliza al PBI per cápita en dólares constantes como una variable que captura el nivel de desarrollo de los países, estos datos provienen del Banco Mundial (BM) y forman parte de los Indicadores de Desarrollo Mundial. La flexibilidad (o complementariamente la rigidez) en el mercado laboral

\footnotetext{
${ }^{\dagger}$ En esta literatura, la informalidad tiende a generar un efecto negativo sobre la productividad y el crecimiento económico (Lopez y Servén, 2009; Perry et al., 2007; Loayza, 2008), impacto que es enfatizado en economías con regulaciones costosas y servicios públicos deficientes. Entre otras razones, la literatura señala que la informalidad desincentivaría el crecimiento de la productividad y de la economía porque: i) limita el acceso al crédito formal, ii) restringe el acceso al comercio exterior y la diversificación de la oferta exportable; iii) promueve un sector empresarial atomizado ((miedo a crecer"); iv) genera congestión en la infraestructura y servicios de uso público; v) fomenta la distribución ineficiente de recursos productivos; vi) restringe la calidad de infraestructura y servicios públicos (e.g. educación, salud, seguridad pública, sistema legal) dada la menor recaudación tributaria; y vii) reduce el grado de competencia en la economía al introducir competencia desleal.
} 
se aproxima mediante las series de flexibilidad laboral que componen el índice de competitividad global del World Economic Forum (WEF). Específicamente se consideran dos indicadores que están disponibles con frecuencia anual entre 2006 y 2019. La primera serie es la facilidad para contratar y despedir trabajadores, y la segunda es la flexibilidad para la determinación de salarios, ambas series toman valores entre 1 y 7 , siendo 7 el valor máximo y que corresponde a la económica más flexible en términos laborales y 1 a la economía con altos niveles de rigidez laboral. Así, por ejemplo, si el primer indicador toma el valor de 7 la economía tiene las prácticas más flexibles para contratar y despedir trabajadores, y si toma el valor de 1 en esta economía la contratación y despido de trabajadores es muy rigida. La facilidad para despedir y contratar en promedio es 3,6 y la facilidad para la determinación de salarios en promedio es 4,7 .

Otro indicador de relevancia es la productividad. Para este caso se considera a la Productividad Total de Factores (PTF) estimada por Penn World Tables 9.1 (PWT). Los datos de PWT corresponden a un indicador de productividad relevante para análisis de bienestar, pero expresado como índice relativo a la productividad de Estados Unidos de América (USA). Sin embargo, en este estudio se requiere un indicador de productividad en niveles y en términos reales para una fácil interpretación de los resultados. Para obtener este indicador se usa de manera conjunta los datos de la PTF en niveles estimados por The Conference Board para USA y el índice de PTF de Penn World Tables que expresa la PTF como índice respecto a USA, el indicador de PTF se obtiene al multiplicar la PTF en niveles de USA de TCB con la PTF de cada país (relativo a USA) estimado por PWT.

Los datos utilizados en las estimaciones corresponden al periodo 2007 - 2017, estos datos o la correlación agregada entre informalidad laboral, actividad económica y los indicadores de rigidez laboral por países se presentan en los Gráficos 1 al 4. Otra característica que se destaca de los datos es la tendencia hacia una mejora de la actividad económica. Similarmente, la informalidad laboral ha mostrado una tendencia decreciente, especialmente en años recientes cuando pasó de 34\% en 2012 a 30\% en 2017.

\section{Resultados}

\section{Informalidad laboral y flexibilidad laboral}

El análisis econométrico considera al rezago del PBI per cápita (y la productividad) como una variable de estudio, que al estar rezagada debería ayudar a controlar por los potenciales problemas de ineficiencia de estimadores que se podría presenar si se incorpora al $\mathrm{PBI}$ del mismo periodo como variable explicativa. Una consideración a tomar en cuenta es que las variables de flexibilidad laboral son consideradas como variables institucionales que tienen poca dinámica, razón por la cual se consideran variables exógenas dentro del análisis de regresión (estas variables también se rezagan un periodo en las estimaciones). La razón fundamental es que el grado de rigidez laboral que existe en los países es el resultado del arreglo institucional y/o legal. En el caso peruano esto podría ser fácilmente ilustrado por los cambios legales que han configurado un contexto de alta estabilidad laboral y por lo tanto alta rigidez para el despido de trabajadores (ver Tablas 23 4).
TABLA 2

TABLA 3

TABLA 4

La ecuación 1 se estima por mínimos cuadrados ordinarios robustos y por efectos fijos. En este procedimiento los indicadores de flexibilidad laboral se consideran exógenos, ${ }^{\S}$ y para el caso del PBI se considera un rezago y de este modo se reduce los potenciales problemas de inconsistencia de los estimadores que han sido documentados por la literatura. Por otra parte, la estimación por el método de efectos fijos ayuda a controlar por las características idiosincráticas de los países considerados y que no cambian en el periodo de estudio.

En términos generales ambos métodos reportan una relación negativa entre la flexibilidad laboral e informalidad laboral, siendo la magnitud de esta relación pequeña. La elasticidad de la flexibilidad laboral es menor a 0,1 en valor absoluto. Con esta evidencia se puede afirmar que ante incrementos de la rigidez laboral (o alternativamente una menor flexibilidad laboral) es de esperar que la informalidad laboral se incremente moderadamente.

La estimación que considera solo a la rigidez laboral como variable explicativa reporta una alta elasticidad de la flexibilidad laboral (columnas 1 y 6); sin embargo, al incluir el nivel de actividad como variable explicativa adicional la magnitud de esta relación se reduce ligeramente, tomando relevancia la actividad económica como variable que esta correlacionada con la informalidad. Este último resultado ha sido documentado por diversos estudios."

La flexibilidad laboral tiene efectos en la reducción de la informalidad que son menores respecto a los efectos del crecimiento económico. Los coeficientes del PBI y de la flexibilidad laboral son negativos y se resalta que el coeficiente del PBI es mayor al coeficiente de la flexibilidad laboral (en valor absoluto), esto se observa considerando los dos tipos de flexibilidad laboral. Notar que en la representación econométrica utilizada se pueden

¥Al incluir al PBI per cápita como variable explicativa se está capturando indirectamente al efecto de la productividad en la informalidad laboral. Esto bajo la premisa de que la productividad en las economías desarrolladas (que son poco informales) está altamente correlacionada con sus altos niveles de PBI per cápita. En la sección siguiente se incluye la productividad y se verifica que la importancia de la flexibilidad laboral se mantiene en esta nueva formulación que incluye productividad en lugar del PBI per cápita como variable que explica a la informalidad laboral.

$\S$ Los indicadores de flexibilidad laboral considerados tienen alta persistencia a lo largo del tiempo. Así, por ejemplo, al relacionar los indicadores de flexibilidad con sus rezagos se tienen que la elasticidad respectiva es cercana a $1 \quad(0,97$ para la facilidad para contratar y despedir y 0,96 para la flexibilidad en la determinación de salarios). Esta regularidad sugiere que los indicadores de flexibilidad tienen alta inercia y los rezagos de estos indicadores son instrumentos válidos de sus respectivos valores corrientes. Esto último sugiere que al usar los valores corrientes de la flexibilidad laboral se reportaría estimadores consistentes de la elasticidad informalidad \{ flexibilidad laboral.

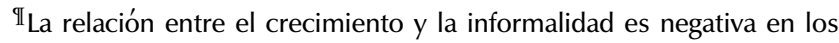
datos, lo cual es similar a los resultados de Loayza y Rigolini (2006) y Céspedes (2015) para el caso peruano. Los resultados de este reporte sugieren que los argumentos sostenidos en Loayza se mantienen con datos recientes. 
interpretar como pseudo elasticidades al tener logaritmos como variables explicativas, por esta razón se concluye que la sensibilidad de la informalidad respecto al PBI es mayor en comparación a la sensibilidad al considerar la flexibilidad laboral.

Un resultado que se destaca es que la relación entre la informalidad y la flexibilidad del mercado laboral es no lineal. Los dos tipos de no linealidad considerados son relevantes, es decir el termino cuadrático de la flexibilidad laboral y el término de interacción de la flexibilidad laboral con el PBI per cápita rezagado. Aunque al incluir estos términos de no linealidad en la regresión el coeficiente asociado al efecto contemporáneo de la flexibilidad se convierte en no significativo, con lo cual se podría argumentar que la flexibilidad tiene efectos que son especificos al tipo de países.

Una interpretación que se le puede dar a esta no linealidad es que la flexibilidad para contratar y despedir afecta a la informalidad según el nivel de rigidez laboral que se encuentre en los países. Incrementar la rigidez laboral en económicas con baja rigidez laboral puede incrementar la informalidad laboral ligeramente, mientras que en las económicas que se caracterizan por tener alta rigidez laboral los efectos de una mayor rigidez laboral son mayores en términos de informalidad.

En otros términos, lo que este resultado sugiere es que niveles altos de rigidez son más costosos en términos de informalidad que niveles bajos de rigidez. La contraparte de este argumento es que debería ser más fácil reducir la informalidad en términos absolutos en economías con altos niveles de rigidez laboral, mientas que esta facilidad se reduce conforme los niveles de rigidez se reducen.

\section{Informalidad laboral y productividad}

Uno de los argumentos de amplio uso en las políticas de reducción de la informalidad laboral es mediante mejoras en la productividad de los trabajadores. Bajo este argumento se sostiene que los bajos niveles de productividad del sector informal constituye una de las limitaciones mayores a las políticas de reducción de informalidad. Así, por ejemplo, según algunas evidencias para las economías en desarrollo la productividad del sector formal es 2,5 veces la productividad del sector informal. Para incorpora la productividad como determinante de la informalidad laboral se utiliza una extensión de la ecuación 1 que considera a un indicador de la productividad $\left(P T F_{(i t-1)}\right)$ como se indica en la siguiente ecuación:

$$
\text { Informalidad }_{i t}=\alpha+\beta \text { Flex }_{i t}+\theta \text { Flex }_{i} t \times P T F_{i t-1}+\delta X_{i t}+v_{i t}
$$

La ecuación 2 se estima siguiendo el mismo procedimiento utilizado para la ecuación 1 . Un resultado que se destaca es que la productividad esta significativamente correlacionado con la informalidad laboral, lo cual es consistente con la relación anteriormente documentada y con la evidencia internacional disponible. Segundo, la productividad tiene una elasticidad sobre la informalidad que es superior a la elasticidad encontradas cuando se utilizó el PBI per cápita. Un segundo aspecto es que los coeficientes relacionados con los indicadores de flexibilidad laboral cambian ligeramente al utilizar la PTF en lugar del PBI per cápita, lo cual se podría relacionar lo cual sugiere cierta estabilidad en las estimaciones efectuadas.

Un aspecto adicional que se destaca de los resultados se refiere a la magnitud relativa de las elasticidades de la productividad y de la flexibilidad laboral respecto a la informalidad. La introducción conjunta de los indicadores de productividad y flexibilidad laboral en la ecuación de informalidad captura estas dos elasticidades de manera simultánea, que luego de ser estimadas, nos permite sugerir que la productividad podría ser varias veces más eficiente reduciendo la informalidad en comparación a la flexibilidad laboral. Esta afirmación se desprende al comparar las elasticidades que se muestran en el Cuadro 3.

Asimismo, se debe destacar que este resultado tiene implicancias muy relevantes desde la perspectiva de las políticas de reducción de la informalidad, específicamente nos permite discriminar entre las políticas basadas en mejoras de las mejoras directas de la productividad y entre las medidas de política concernientes a la flexibilización laboral, bajo esta premisa las políticas basadas en mejoras en la productividad deberían ser más eficientes en su objetivo de reducir la informalidad.

\section{Limitaciones}

Este estudio constituye una aproximación de la relación promedio entre la productividad, la informalidad y las rigideces del mercado laboral. Se debe destacar que este análisis agregado puede constituir una importante limitación de este estudio al no identificar adecuadamente el mecanismo microeconómico por el cual interactúan, por ejemplo, los indicadores de rigidez laboral y la informalidad. Otro aspecto que se debe destacar como limitación del estudio es el uso de los indicadores de informalidad y de rigideces laborales, estos podrían no capturar adecuadamente las características idiosincráticas de la regulación laboral y de la informalidad en cada región.

Por ejemplo, el indicador de informalidad es el porcentaje de autoempleos en cada país, este indicador es una aproximación de la informalidad que se toma por la aun escasez de indicadores anuales de suficiente cobertura geográfica y temporal. Asimismo, los indicadores de rigidez laboral considerados (rigidez de contratación y despido y rigidez salarial) son indicadores agregados de las fricciones laborales en cada país, este indicador de la WEF representa un promedio de cada país y no logra capturar las heterogeneidades de la regulación que enfrentan los diferentes sectores y empresas de cada país.

Finalmente, se debe recalcar que el enfoque de este estudio es básicamente capturar las tendencias y/o promedios de la relación de la informalidad con la regulación laboral, este promedio creemos constituye un aspecto que se debe destacar.

\section{Conclusiones}

La alta informalidad laboral constituye una de las características estructurales de las economías en desarrollo. Paralelamente, la poca reducción de la informalidad durante las últimas décadas sugiere que la informalidad ha reaccionado lentamente frente a los episodios de alto crecimiento económico que ha caracterizado a estas economías durante las últimas décadas. En este contexto 
una pregunta recurrente en las económicas en desarrollo con alta informalidad se refiere a la capacidad de reducción de la informalidad que tiene el crecimiento económico, a la capacidad que tiene las mejoras en la productividad, $\mathrm{o}$ al rol de las políticas de flexibilización laboral.

La literatura ha explorado también la posibilidad de exista complementariedad entre las políticas de mejoras en productividad y las políticas de mejoras en la flexibilización laboral sobre sus efectos en la informalidad. En este documento se estudia el rol de la productividad y de la flexibilidad laboral, así como a la complementariedad entre ellos, como elementos que pueden afectar a la informalidad laboral.

Este estudio se construye sobre la literatura de determinantes de la informalidad a nivel agregado y por países (Loayza y Rigolini, 2006). Para este fin se utiliza un enfoque empírico considerando a la productividad, estimada por PWT, y al autoempleo, reportado por la OIT como indicador de informalidad laboral, si bien esta última puede ser una limitación al ser un indicador que captura parcialmente la informalidad, la alta correlación que existe entre el autoempleo y la informalidad sugiere que esta limitación podría no ser tan importante. Asimismo, se utiliza indicadores de flexibilidad laboral y salarial de la WEF, estos indicadores capturan el grado de rigidez laboral de las economías en consideración.

Entre los resultados se destaca que la productividad y la flexibilidad laboral afectan a la informalidad laboral de manera negativa, con lo cual las políticas que apuntan hacia una mayor flexibilidad laboral y las que inducen una mayor productividad se relacionan con menores niveles de informalidad laboral. Si bien el signo de las correlaciones consistente con los argumentos anteriores, sin embargo, la magnitud de estas correlaciones sugiere que las elasticidades son bajas, con lo cual se requeriría mejoras mayores de las políticas de productividad y de flexibilidad laboral par que la informalidad pueda mejorar sustancialmente mediante esos dos canales. Por lo tanto, la poca dinámica de la informalidad en el periodo estudiado en un contexto de alto crecimiento económico y de productividad es consistente con los resultados reportados.

Otro aspecto que se discute es sobre la eficiencia relativa que tiene las políticas de mejoras en productividad en comparación con las políticas de flexibilización laboral. En este documento se muestra que la productividad es más efectiva en su capacidad de reducción de la informalidad laboral en comparación a la flexibilidad laboral. Este resultado es destacable pues las evidencias sugieren que la productividad, en gran parte de las economías en desarrollo, es muy baja y por lo tanto al haber espacio de mejoras se podría mejorar los niveles de informalidad sustancialmente.

Finalmente, se muestra que las políticas de flexibilización laboral pueden tener efectos no lineales en la informalidad, esto al reportarse que en las economías con baja flexibilidad laboral una mayor flexibilización laboral tendría mayores efectos en reducir la informalidad laboral, mientras que en economías con alta flexibilidad este tipo de políticas de flexibilización tendrian menores efectos en términos de reducción de informalidad.

\section{Agradecimientos}

Los autores agradecen al apoyo de la Escuela Profesional de Economía y del Instituto de Investigación de la Facultad de Ciencias Contables y Económicas y Financieras de la Universidad de San Martín de Porres.

\section{Referencias}

Almeida, R. and P. Carneiro (2009). Enforcement of labor regulation and firm size. Journal of Comparative Economics, 37, 28-46

Almeida, Rita, and Pedro Carneiro (2012). Enforcement of labor regulation and informality. American Economic Journal: Applied Economics, 4(3), 64-89.

Céspedes, N. (2015), Crecer no es Suficiente para Reducir la Informalidad, Working Papers 2015-005, Banco Central de Reserva del Perú.

Deakin, S. and P. Sarkar (2008). Assessing the Long-run Economic Impact of Labour Law Systems: A Theoretical Reappraisal and Analysis of New Time Series Data. Industrial Relations Journal, 39(6), 453-487.

De Andrade, Gustavo Henrique, Miriam Bruhn, and David McKenzie (2016). A helping hand or the long arm of the law? Experimental evidence on what governments can do to formalize firms. The World Bank Economic Review, $30(1), 24-54$

De Soto, H. (1989), The other path, New York, Harper and Row.

Fajnzylber, Pablo, William F Maloney, and Gabriel V MontesRojas (2011). Does formality improve microfirm performance? Evidence from the Brazilian SIMPLES program. Journal of Development Economics, 94(2), 262276.

Feldmann, H. (2009). The unemployment effects of labor regulation around the world. Journal of Comparative Economics, 37(1), 76-90.

ILO(2013) The informal economy and decent work: a policy resource guide, supporting transitions to formality / International Labour Office.

International Labor Office y World Trade Organization (2009), Globalization and Informal Jobs in Developing Countries, International Labour Office and the Secretary of the World Trade Organization.

Loayza, N. (2008), Causas y consecuencias de la informalidad en Perú, Revista Estudios Económicos, BCRP 15, 43-64.

Loayza, N. y J. Rigolini (2006), Informality Trends and Cycles, Policy Research Working Paper 4078. Washington, DC, United States: World Bank.

Lopez, H. y L. Servén (2009), Too poor to grow, Policy Research Working Paper 5012, World Bank.

Monteiro, J. and Assuncao, J. (2012). Coming out of the shadows? Estimating the impact of bureaucracy simplification and tax cut on formality in Brazilian microenterprises. Journal of Development Economics, 99(1), 105-115.

OECD (2004), Informal Employment and Promoting the Transition to a Salaried Economy, in OECD Employment Outlook, OECD Publishing. 
Perry, G., W. Maloney, O. Arias, P. Fajnzylber, A. Mason y J. Saavedra-Chanduvi (2007), Informality, exit and exclusion (Washington, DC, World Bank).

Schneider, F. (1994), Measuring the Size of the Shadow Economy: Can the Causes be found and the Obstacles be Overcome? in Brandstatter, H. and W. Guth (editors), Essays on Economic Psychology pp. 193-212.

Schneider, F. (2005), Shadow economies of 145 countries all over the world What do we really Know?, Working Paper 13 (Basel, Center for Research in Economics, Management

Rocha, R., Gabriel U. and Rachter,L. (2018). Do lower taxes reduce informality? Evidence from Brazil. Journal of Development Economics, 134, 28-49.

World Bank (1990). World Development Report \{ Poverty, New York: Oxford University Press.

\section{FIGURAS Y CUADROS}

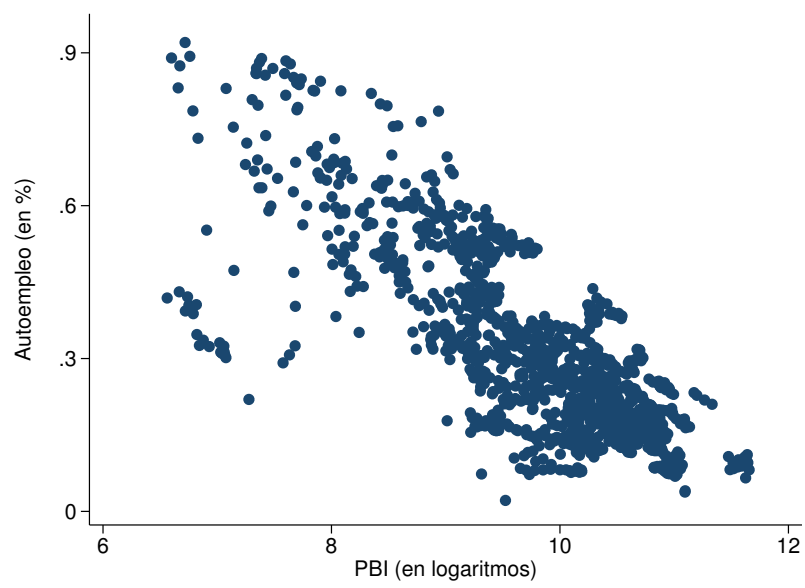

Nota: Los datos corresponden al periodo 2007 - 2017. El PBI per capita esta en terminos reales y se expresa en logaritmos. El Autoempleo se expresa en porcentajes. Fuente: OIT y WB.

Figura 1. Informalidad y PBI Per capita

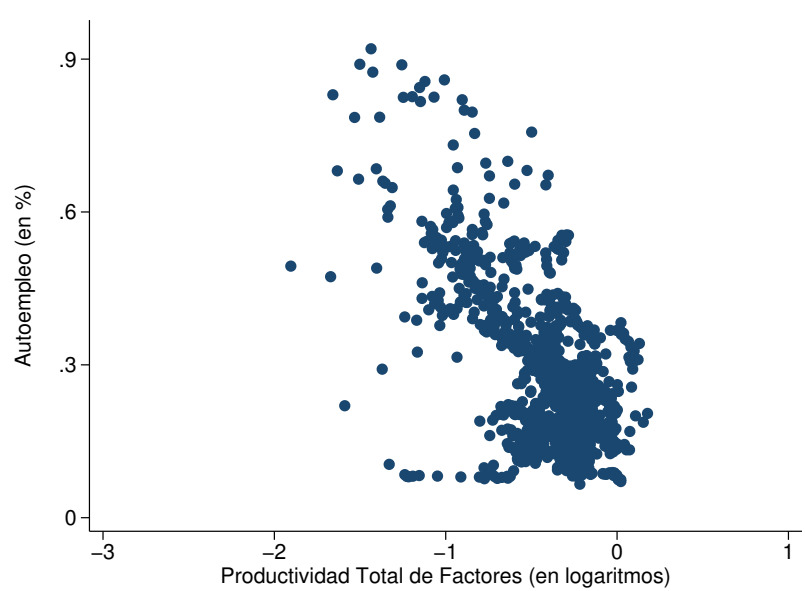

Nota: Los datos corresponden al periodo 2007 - 2017. La Productividad total de factores esta en logaritmos. El Autoempleo se expresa en porcentajes Fuente: ILO, TCB, PWT y WB.

Figura 2. Informalidad y productividad total de factores 


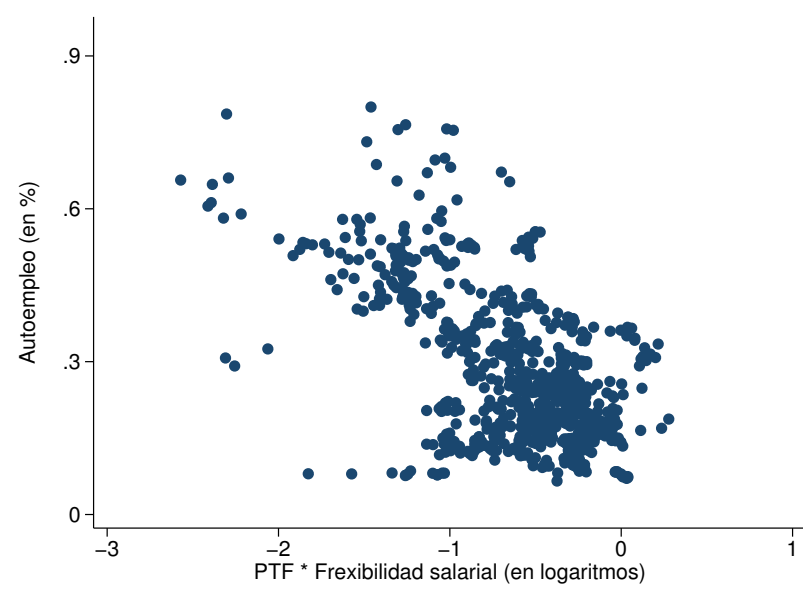

Nota: Los datos corresponden al periodo 2007-2017 para 89 parses. La facilidad para contratar y despedir corresponde a la interaccion del indicador de facilidad para contratar y despedir de la WEF y el PBI per capita del WB. Fuente: OIT y WEF.

Figura 3. Informalidad y facilidad para contratar y despedir trabajadores
Cuadro 1. Estadısticas descriptivas, 2007-2017

\begin{tabular}{lccccc}
\hline & $\begin{array}{c}\text { No } \\
\text { obs. }\end{array}$ & Promedio & $\begin{array}{c}\text { Desv. } \\
\text { std. }\end{array}$ & Mınimo & Maximo \\
\hline Flexibilidad salarial & 736 & 4.73 & 0.22 & 2.18 & 6.25 \\
Facilidad laboral & 736 & 3.66 & 0.21 & 1.92 & 6.11 \\
Autoempleo & 736 & 27.9 & 0.53 & 6.6 & 85.6 \\
PBI per capita & 736 & 29.5 & 0.77 & 0.89 & 115.4 \\
Productividad (PTF) & 736 & 0.70 & 0.28 & 0.25 & 1.17 \\
& & & & & \\
\hline
\end{tabular}

Nota: Promedio de valores entre 2007 - 2017. La desviacion estandar se calcula a las series en logaritmos. La Flexibilidad salarial corresponde al Indice de flexibilidad para la determinacion de salarios (Indice WEF). La flexibilidad laboral corresponde al Indice de practicas de despido y contratacion de la WEF. El PBI per capita esta en miles de dolares reales de 2011, el autoempleo esta en porcentajes, y la productividad corresponde a la PTF estimada de PWT y TCB. Fuente: OIT, BM, WEF, TCB y PWT.

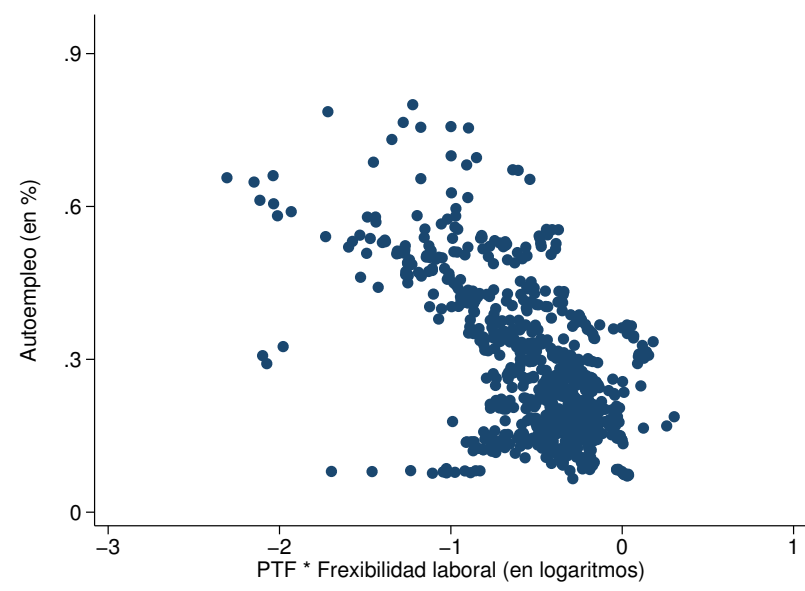

Nota: Los datos corresponden al periodo 2007-2017 para 89 parses. La flexibilidad salarial corresponde a la interaccion del indicador de la flexibilidad en la determinacion de salarios de la WEF y el PBI per capita del WB. Fuente: OIT y WEF.

Figura 4. Informalidad y flexibilidad salarial
Cuadro 2. Estimacion de ecuacion de informalidad laboral

\begin{tabular}{lrrrrr}
\hline & \multicolumn{1}{c}{$(1)$} & \multicolumn{1}{c}{$(3)$} & \multicolumn{1}{c}{$(4)$} & \multicolumn{1}{c}{$(5)$} \\
\hline & & & & & \\
$\log (\mathrm{PBI}(-1))$ & & $-0.121^{* * *}$ & $-0.121^{* * *}$ & $-0.466^{* * *}$ & $-0.317^{* * *}$ \\
Flexibilidad laboral & $-0.045^{*}$ & $-0.027^{*}$ & $(-25.5)$ & $(-22.33)$ & $(-1.72)$ \\
& $(-2.33)$ & $(-1.64)$ & & & 1.23 \\
Flexibilidad laboral ${ }^{2}$ & & $-0.012^{*}$ & & & $(0.82)$ \\
& & $(-2.04)$ & & & \\
Flexibilidad laboral $\times \log (\mathrm{PBI}(-1))$ & & & -0.018 & -0.136 \\
& & & & $(-2.5)$ & $(-0.96)$ \\
Constante & & & & \\
& $0.293^{* * *}$ & $1.504^{* * *}$ & $1.486^{* * *}$ & 3.450 & 1.896 \\
& $(11.84)$ & $(26.96)$ & $(29.09)$ & $(16.93)$ & $(0.97)$ \\
R cuadrado & & & & & \\
No. Obs. & 0.0088 & 0.5376 & 0.5387 & 0.4711 & 0.4722 \\
prob > F & 603 & 603 & 603 & 603 & 603 \\
& 0.000 & 0.000 & 0.000 & 0.000 & 0.000 \\
\hline
\end{tabular}

Nota: Estimado por mınimos cuadrados ordinarios. La flexibilidad laboral se refiere a la facilidad para despedir y contratar de la WEF. Este Indice toma valores entre 1 y 7 , siendo 7 el indicador de maxima facilidad para contratar y despedir y/o mayor flexibilidad laboral. La flexibilidad salarial se refiere a la flexibilidad en la determinacion de salarios; este Indice es estimado anualmente por la WEF y toma valores entre 1 y 7 , siendo 7 la maxima flexibilidad en la determinacion de salarios. $\log (\mathrm{PBI}(-1))$ es el logaritmo natural del PBI rezagado un año. $*, * *, * * *$ representa significancia estadıstica al $10 \%, 5 \%$ y $1 \%$, respectivamente. Estadıstico t entre parentesis.

Fuente: OIT, WB, WEF, TCB y PWT. 
Cuadro 3. Estimacion de ecuacion de informalidad laboral

\begin{tabular}{|c|c|c|c|c|c|}
\hline & (1) & (2) & (3) & (4) & (5) \\
\hline $\log (\operatorname{PTF}(-1))$ & & $\begin{array}{r}-0.204^{* * *} \\
(-8.37)\end{array}$ & $\begin{array}{r}-0.202 * * * \\
(-8.59)\end{array}$ & $\begin{array}{r}-0.537^{* * *} \\
(-5.04)\end{array}$ & $\begin{array}{r}-1.106^{* * *} \\
(-4.36)\end{array}$ \\
\hline Flexibilidad de salarios & $\begin{array}{r}0.003^{*} \\
(0.16)\end{array}$ & $\begin{array}{r}-0.031^{*} \\
(-1.81)\end{array}$ & $\begin{array}{r}0.672^{* * *} \\
(3.72)\end{array}$ & & $\begin{array}{r}0.132 * * \\
(3.14)\end{array}$ \\
\hline Flexibilidad de salarios ${ }^{2}$ & & & $\begin{array}{r}-0.253^{*} \\
(-3.93)\end{array}$ & & \\
\hline Flexibilidad de salarios $\times 1$ & $\operatorname{og}(\operatorname{PTF}(-1))$ & & & $\begin{array}{l}0.212 \\
(3.15)\end{array}$ & $\begin{array}{r}0.584 \\
(3.5)\end{array}$ \\
\hline Constante & $\begin{array}{r}0.232^{* * *} \\
(9.43)\end{array}$ & $\begin{array}{r}0.212^{* * *} \\
(8.55)\end{array}$ & $\begin{array}{r}-0.258^{*} \\
(-2.10)\end{array}$ & $\begin{array}{r}0.163^{* * *} \\
(20.703)\end{array}$ & $\begin{array}{r}-0.037 \\
(0.59)\end{array}$ \\
\hline R cuadrado & 0.0008 & 0.1932 & 0.2153 & 0.2111 & 0.2303 \\
\hline No. Obs. & 603 & 603 & 603 & 603 & 603 \\
\hline prob $>F$ & 0.000 & 0.000 & 0.000 & 0.000 & 0.000 \\
\hline
\end{tabular}

Nota: Estimado por mınimos cuadrados ordinarios. La flexibilidad laboral se refiere a la facilidad para despedir y contratar de la WEF. Este Indice toma valores entre 1 y 7 , siendo 7 el indicador de maxima facilidad para contratar y despedir y/o mayor flexibilidad laboral. La flexibilidad salarial se refiere a la flexibilidad en la determinacion de salarios; este indice es estimado anualmente por la WEF y toma valores entre 1 y 7 , siendo 7 la maxima flexibilidad en la determinacion de salarios. $\log (\mathrm{PBI}(-1))$ es el logaritmo natural del $\mathrm{PBI}$ rezagado un año. $*, * *, * * *$ representa significancia estadıstica al $10 \%, 5 \%$ y $1 \%$, respectivamente. Estadıstico t entre parentesis.

Fuente: OIT, WB, WEF, TCB y PWT.

Cuadro 4. Estimacion de ecuacion de informalidad laboral (Estimador de efectos fijos)

\begin{tabular}{lrrrrr}
\hline & $(1)$ & \multicolumn{1}{c}{$(2)$} & \multicolumn{1}{c}{$(3)$} & \multicolumn{1}{c}{$(4)$} & \multicolumn{1}{c}{$(5)$} \\
\hline & & & & & \\
$\log (\mathrm{PBI}(-1))$ & & $-0.057^{* * *}$ & $-0.057^{* * *}$ & $-0.149^{* * *}$ & $-0.293^{* * *}$ \\
& & $(-6.33)$ & $(-6.36)$ & $(-3.99)$ & $(-4.02)$ \\
Flexibilidad laboral & -0.008 & -0.007 & -0.081 & & $-1.121^{*}$ \\
& $(-1.19)$ & $(-0.99)$ & $(-1.56)$ & & $(-2.30)$ \\
Flexibilidad laboral ${ }^{2}$ & & & $\begin{array}{r}0.031^{* * *} \\
(1.44)\end{array}$ & & \\
Flexibilidad laboral $\times \log (\mathrm{PBI}(-1))$ & & & 0.000 & $0.109^{*}$ \\
& & & & $(0.11)$ & $(2.30)$ \\
& & & & & \\
Constante & $0.267^{* * *}$ & $0.842^{* * *}$ & $0.887^{* * *}$ & 0.017 & $1.504^{*}$ \\
& $(30.39)$ & $(9.10)$ & $(9.08)$ & $(0.04)$ & $(2.01)$ \\
& & & & & \\
R cuadrado & 0.001 & 0.587 & 0.581 & 0.534 & 0.5131 \\
No. Obs. & 603 & 603 & 603 & 603 & 603 \\
prob $>\mathrm{F}$ & 0.000 & 0.000 & 0.000 & 0.000 & 0.000 \\
\hline
\end{tabular}

Nota: La flexibilidad laboral se refiere a la facilidad para despedir y contratar de la WEF. Este indice toma valores entre 1 y 7 , siendo 7 el indicador de maxima facilidad para contratar y despedir y/o mayor flexibilidad laboral. La flexibilidad salarial se refiere a la flexibilidad en la determinacion de salarios; este indice es estimado anualmente por la WEF y toma valores entre 1 y 7 , siendo 7 la maxima flexibilidad en la determinacion de salarios. $\log (\mathrm{PBI}(-1))$ es el logaritmo natural del PBI rezagado un año. $*, * *, * * *$ representa significancia estadıstica al $10 \%$, $5 \%$ y $1 \%$, respectivamente. Estadıstico t entre parentesis.

Fuente: OIT, WB, WEF, TCB y PWT. 